\title{
Analytical validation of serum bone alkaline phosphatase (BAP OSTASE) on Liaison
}

\author{
Etienne Cavalier ${ }^{1, *}$, Eric Rozet ${ }^{2, a}$, Agnès Carlisi ${ }^{1}$, \\ Anne-Catherine Bekaert ${ }^{1}$, Olivier Rousselle ${ }^{1}$, \\ Philippe Hubert ${ }^{2}$, Jean-Paul Chapelle ${ }^{1}$ and \\ Pierre Delanaye ${ }^{3}$ \\ ${ }^{1}$ Department of Clinical Chemistry, University of Liege, \\ University Hospital of Liege, Liege, Belgium \\ ${ }^{2}$ Department of Analytical Chemistry, University of Liege, \\ Liege, Belgium \\ ${ }^{3}$ Department of Nephrology and Dialysis, University of \\ Liege, University Hospital of Liege, Liege, Belgium
}

\begin{abstract}
Background: The goal of this study was to validate the DiaSorin Liaison BAP OSTASE, a new method for measurement of bone alkaline phosphatase (BAP), and to compare this method with the Beckman-Coulter Access Ostase. We also wanted to establish the reference range for BAP in adults and children.

Methods: We determined the precision, functional sensitivity, recovery, linearity and measurement uncertainty, accuracy profile and $\beta$-expectation limits. We defined an adult reference interval using individuals with $25-\mathrm{OH}$ vitamin $\mathrm{D}$ $>80 \mathrm{nmol} / \mathrm{L}$, parathormone $<58 \mathrm{ng} / \mathrm{L}$, and normal calcium, phosphorous and estimated glomerular filtration rate. Each adult subclass (men/non-menopausal women/menopause women) contained 120 individuals. We also determined the 2.5th and 97.5th percentiles from a population of 450 children, stratified according to age and gender.

Results: The results of the validation showed: precision $<6 \%$, functional sensitivity $<0.74 \mu \mathrm{g} / \mathrm{L}$, mean recovery $98.8 \pm 4.2 \%$ and good linearity. Relative uncertainty ranged from $9.0 \%$ to $12.9 \%$, and the risk of one result falling out of the $\pm 15 \%$ acceptance limits was $<5 \%$ for concentrations between 7 and $94 \mu \mathrm{g} / \mathrm{L}$. The Bland-Altman plot showed no systematic bias between the two methods. In adults, we did not find any statistical difference between the different subclasses. The upper limit of normality observed in the entire population $(\mathrm{n}=360)$ was $21.3 \mu \mathrm{g} / \mathrm{L}(90 \% \mathrm{CI}: 18.3-24.2$ $\mu \mathrm{g} / \mathrm{L})$.
\end{abstract}

${ }^{\mathrm{a}}$ F.R.S.-FNRS Postdoctoral Researcher (Belgium). *Corresponding author: Dr. Etienne Cavalier, Department of Clinical Chemistry, University Hospital of Liège, Domaine du Sart-Tilman, 4000 Liège, Belgium

Phone: + 324 3667692, Fax: + 3243667691 ,

E-mail: Etienne.cavalier@chu.ulg.ac.be

Received August 1, 2009; accepted September 2, 2009;

previously published online November 30, 2009
Conclusions: The Liaison BAP OSTASE is a robust method, and is completely validated between 7 and $93 \mu \mathrm{g} / \mathrm{L}$ : in this range, $95 \%$ of the values obtained will be within $\pm 15 \%$ of the true value.

Clin Chem Lab Med 2010;48:67-72.

Keywords: analytical validation; bone alkaline phosphatase; measurement uncertainty; reference range.

\section{Introduction}

Bone alkaline phosphatase (BAP) was discovered in 1923 by Dr. Robert Robinson from the Lister Institute (1). BAP is one isoform of the alkaline phosphatases, which originates from many tissues, but primarily the liver and bone. BAP is a tetrameric glycoprotein attached to the osteoblast membrane by a carboxy-terminal glycan-phosphatidylinositol anchor. The action of a phospholipase releases BAP as a homodimer from the membrane into the circulation (2). Once in the blood stream, the half-life of BAP is relatively long (1-2 days), which most likely contributes to its low diurnal and biological variability (3). The exact function of BAP remains unknown. However, it has been suggested that it might play a role in mineralisation of newly formed bone. Indeed, patients suffering from hypophosphatasia, a rare inborn error of metabolism caused by low activity of the tissue-non-specific isoenzyme of alkaline phosphatase, present with severe osteomalacia. BAP is a useful marker in rare diseases where its concentration is particularly high such as Paget's disease of bone or tumour-induced osteomalacia. However, it is most often used as a marker of bone remodelling during follow-up of osteoporosis. Since it is cleared by the liver (4) and therefore not affected by kidney disease, BAP also represents an interesting alternative to parathyroid hormone (PTH) in the follow-up of patients suffering from renal osteodystrophy. Commercially available immunoassays have been developed for measurement of BAP. These assays measure either the activity or the mass of the enzyme. The goal of this study was to evaluate the analytical performance of the BAP OSTASE (DiaSorin, Stillwater, MN, USA). This method has been made available recently on the Liaison platform. We wished to compare the performance of this kit with the Beckman-Coulter Access (Beckman-Coulter, Brea, CA, USA). We also defined the reference interval of this parameter in a well described "normal" adult population and in children. 
Table 1 Precision observed in three serum controls.

\begin{tabular}{|c|c|c|c|c|c|c|}
\hline \multirow[t]{2}{*}{ Control } & \multirow[t]{2}{*}{$\mathrm{n}$} & \multirow[t]{2}{*}{ Mean, $\mu \mathrm{g} / \mathrm{L}$} & \multicolumn{2}{|c|}{ Within-run } & \multicolumn{2}{|l|}{ Total } \\
\hline & & & SD & $\mathrm{CV}, \%$ & SD & $\mathrm{CV}, \%$ \\
\hline 1 & 15 & 15.87 & 0.62 & 3.9 & 0.76 & 4.7 \\
\hline 2 & 15 & 21.51 & 0.56 & 2.6 & 1.05 & 4.9 \\
\hline 3 & 15 & 31.14 & 1.50 & 4.8 & 1.81 & 5.8 \\
\hline
\end{tabular}

Table 2 Measurement uncertainty observed in 6 control samples.

\begin{tabular}{llllll}
\hline $\begin{array}{l}\text { Control } \\
\text { level }\end{array}$ & $\begin{array}{l}\text { Mean, } \\
\mu \mathrm{g} / \mathrm{L}\end{array}$ & $\begin{array}{l}\text { Uncertainty, } \\
\mu \mathrm{g} / \mathrm{L}\end{array}$ & $\begin{array}{l}\text { Relative } \\
\text { uncertainty, } \%\end{array}$ & $\begin{array}{l}\beta \text {-Expectation } \\
\text { tolerance limit, } \mu \mathrm{g} / \mathrm{L}\end{array}$ & $\begin{array}{l}\text { Relative } \\
\beta \text {-expectation } \\
\text { tolerance limit, } \%\end{array}$ \\
\hline 1 & 6.20 & 0.78 & 12.63 & {$[5.243,7.157]$} & {$[-15.44,15.44]$} \\
2 & 15.87 & 1.62 & 10.19 & {$[14.06,17.68]$} & {$[-11.39,11.41]$} \\
3 & 21.51 & 2.27 & 10.52 & {$[18.73,24.29]$} & {$[-12.90,12.93]$} \\
4 & 31.14 & 3.81 & 12.22 & {$[26.93,35.35]$} & {$[-13.52,13.52]$} \\
5 & 51.60 & 4.65 & 9.00 & {$[46.60,56.60]$} & {$[-9.692,9.692]$} \\
{$[-14.72,14.72]$} & 0.623 \\
6 & 93.90 & 12.14 & 12.93 & {$[80.08,107.7]$} & 6.057 \\
\hline
\end{tabular}

\section{Materials and methods}

\section{Analytical methods}

The Liaison BAP OSTASE is a two-site sandwich immunoassay that utilises two purified mouse monoclonal antibodies for capture and detection of BAP. Serum is incubated with paramagnetic particles coated with BAP monoclonal antibody (Mab). After incubation, a second mouse Mab conjugated to isoluminol is added. After another short incubation, a chemiluminescent reaction occurs following addition of the starter reagent. The light signal is proportional to the concentration of BAP present in the sample. The Liaison BAP OSTASE has been calibrated against the BeckmanCoulter Access Ostase.

The Beckman-Coulter Access Ostase is a chemiluminescent immunoenzymatic one-step assay based on capture of the analyte by a mouse Mab specific to BAP and fixation of the complex by paramagnetic particles coated with goat ant-mouse polyclonal antibody. Following the formation of the solid phase-capture Mab-BAP, particles are separated in a magnetic field and washed. A chemiluminescent substrate for alkaline phosphatase is added and fluorescence is measured with a luminometer. Light production is directly proportional to the amount of BAP captured by the Mab. The Access Ostase is an activity-based immunoassay, whereas the Liaison BAP OSTASE is mass-based. The Access Ostase has been matched to the calibration established with the Tandem-R-Ostase IRMA, a mass-based radioimmunoassay.

\section{Statistical software}

We used Medcalc (Mariakerke, Belgium) and e-noval (Arlenda, Liège, Belgium) software for statistical evaluation of the results.

\section{Samples}

We validated the BAP Liaison assay using serum samples.

\section{Validation protocol}

For validation, we evaluated the precision in accordance with a modified protocol based on CLSI EP-5A2 (5). Three homemade serum controls were assayed in triplicate once per day for 5 days. The limit of quantification was defined as the lowest value giving an inter-assay coefficient of variation of $20 \%$, determined over 10 days. Linearity was evaluated in accordance with CLSI EP-6A (6) and recovery was determined according to CLSI EP-6P (7). Finally, we evaluated measurement uncertainty, accuracy and the $\beta$ expectation limits by analysis of six serum pools in triplicate over 5 days.

Measurement uncertainty characterizes the dispersion of the values around the true value and $\beta$-expectation tolerance limits with $\beta=0.95$ are the upper and lower values in-between which each future measurements of the same level has a probability of $95 \%$ to be found $(8,9)$. Accuracy characterises the performance of the method and refers to the closeness of agreement between a result obtained with the method and results obtained with the reference method (10). Since no certified material is available for BAP, we used the mean of each pool as the expected value.

\section{Comparison with the Beckman-Coulter Access}

We compared the results obtained with the two methods in 83 consecutive samples from our daily routine using Bland-Altman analysis (11).

\section{Establishment of the reference range}

We defined our adult normal population as healthy individuals presenting with PTH concentrations $<58 \mathrm{ng} / \mathrm{L}$ (on DiaSorin Liaison), $25-\mathrm{OH}$ vitamin $\mathrm{D}[25(\mathrm{OH}) \mathrm{D}]>80 \mathrm{nmol} / \mathrm{L}$ (DiaSorin Liaison), an estimated glomerular filtration rate $>60 \mathrm{~mL} / \mathrm{min} / 1.73 \mathrm{~m}^{2}$ [isotope dilution mass spectrometry (IDMS) traceable creatinine compensated Jaffé method from Roche Diagnostics on Modular P, Mannheim, Germany], and calcium and phosphorus concentrations falling within the laboratory's reference range (also on Modular P). We rejected 


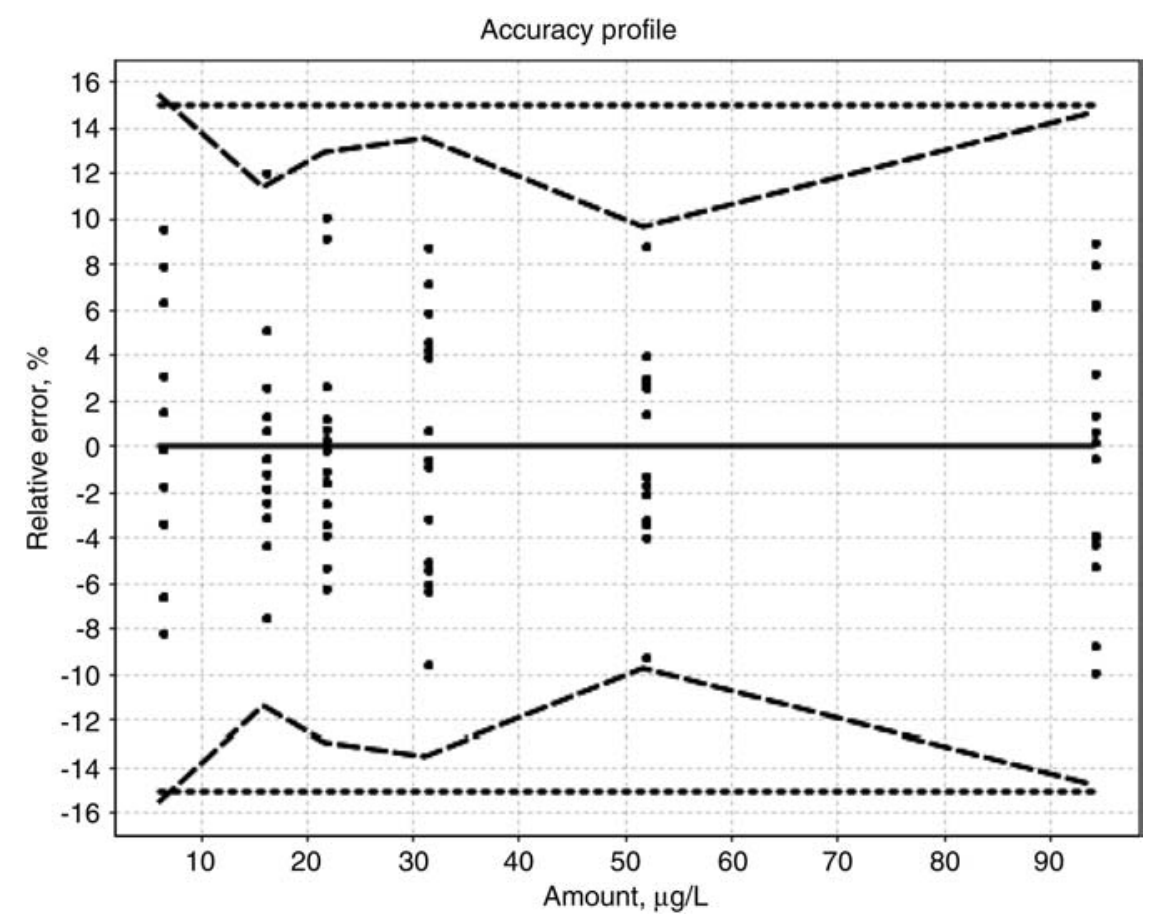

Figure 1 Accuracy profile of the DiaSorin Liaison BAP OSTASE.

When the $\beta$-expectation limits (broken lines) are comprised between the maximum total allowable error (determined to be $\pm 15 \%$ ), the method is considered as valid.

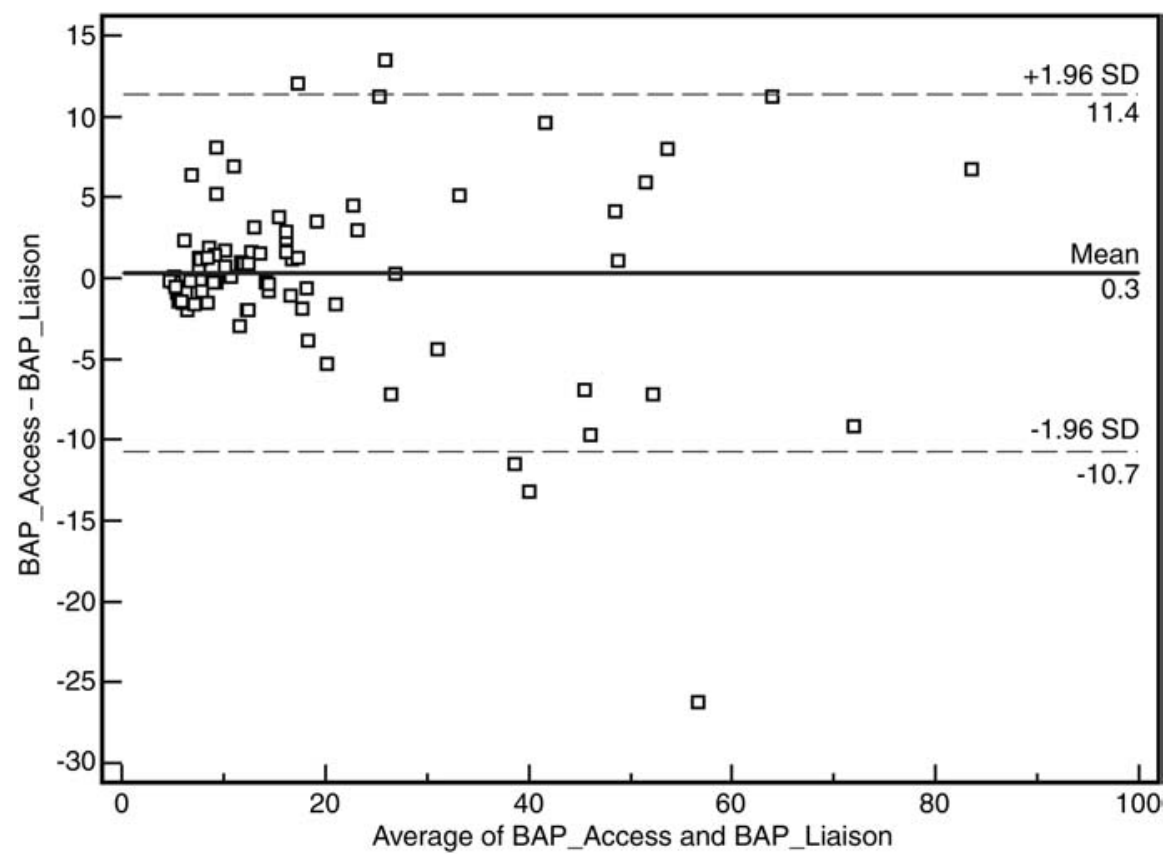

Figure 2 Bland-Altman plot for BAP measured in 83 patients with the Liaison BAP OSTASE and the Beckman-Coulter Access Ostase. Results given in $\mu \mathrm{g} / \mathrm{L}$.

any individual who presented with C-telopepide of type I collagen concentrations (determined with the $\beta$-Crosslaps kit of Roche Diagnostics on Elecsys) higher than the expected reference range. All samples were collected between July and December 2008 and stored for a maximum of 1 month at $-20^{\circ} \mathrm{C}$ before measurement of BAP.
We divided our population in three sub-groups of 120 individuals: males, non-menopause females and menopause females. We used the Kolmogorov-Smirnov test to determine if the population was Gaussian, and non-parametric methods for non-Gaussian distributions. We took the upper $95 \%$ as the upper limit of normality. 
We also measured BAP in children. The children tested had undergone collection of blood for allergy testing. None were suffering from bone disorders, delayed puberty or growth deficiency. Each age category (from 0 to 18 years, 2 years by 2 years) and gender comprised 25 individuals.

\section{Results}

\section{Analytical performance}

The results of the precision evaluation are shown in Table 1. As seen in Table 1, repeatability did not exceed $5 \%$ and the intermediate precision was $6 \%$ at a concentration range of 15-31 $\mu \mathrm{g} / \mathrm{L}$. The functional sensitivity was $0.74 \mu \mathrm{g} / \mathrm{L}$ and mean recovery was $98.8 \% \pm 4.2 \%$. The manufacturer states that the kit is linear up to $120 \mu \mathrm{g} / \mathrm{L}$. By diluting two high samples (80 and $115 \mu \mathrm{g} / \mathrm{L}$ ), we found that the method was linear up to a $1 / 10$ dilution.

Measurement uncertainty and $\beta$-expectation tolerance limits observed using the six pools are presented in Table 2. Measurement uncertainty was between $9.0 \%$ and $12.9 \%$. The $\beta$-expectation tolerance intervals were computed at each concentration with a probability of $\beta=95 \%$. Stated differently, on average, $95 \%$ of the future results generated by this method will be included in the established tolerance intervals. The method for establishing tolerance intervals is predictive. The method will provide accurate results if the $95 \%$ $\beta$-expectation tolerance interval at each concentration is included in the acceptance limits established at $\pm 15 \%$ [according to (12)]. Figure 1 illustrates this using an accuracy profile. As shown on Figure 1, the method provides accurate results between 7 and $94 \mu \mathrm{g} / \mathrm{L}$. These values represent the lower and upper quantitation limits, respectively, and define
Table 3 Description (mean \pm SD) of the adult population used for establishing the reference range.

\begin{tabular}{llll}
\hline & Male & $\begin{array}{l}\text { Non-menopause } \\
\text { female }\end{array}$ & $\begin{array}{l}\text { Menopause } \\
\text { female }\end{array}$ \\
\hline $\mathrm{n}$ & 120 & 120 & 120 \\
Age, years & $57.2 \pm 16.8$ & $31.3 \pm 5.3$ & $67.3 \pm 7.7$ \\
25(OH)D, nmol/L & $94 \pm 15$ & $102 \pm 25$ & $96 \pm 17$ \\
$\mathrm{PTH}, \mathrm{ng} / \mathrm{L}$ & $40 \pm 10$ & $35 \pm 10$ & $40 \pm 10$ \\
\hline
\end{tabular}

the range of the method. Future results are assumed to be within $\pm 15 \%$ of the actual value with a probability of $95 \%$.

\section{Comparison with Beckman-Coulter Access Ostase}

Figure 2 shows the Bland-Altman plot. The values ranged from 3.7 to $80.2 \mu \mathrm{g} / \mathrm{L}$ and there was no systematic bias between the two methods (mean difference $=0.3 \mu \mathrm{g} / \mathrm{L}$ ). The standard deviation (SD) of the differences was $5.7 \mu \mathrm{g} / \mathrm{L}$. We did not find any significant difference using the Wilcoxon test [median (95\% CI): Liaison: $12.8 \mu \mathrm{g} / \mathrm{L}(9.4-21.4)$ and Access: $14 \mu \mathrm{g} / \mathrm{L}$ (10.8-17.2)]. The regression between the two methods is Access $=0.9123 \times$ Liaison $+2.0025\left(\mathrm{R}^{2}=\right.$ 0.9006, $\mathrm{p}<0.001)$.

\section{Values observed in the population}

Our selected population for establishing an adult range showed a mean $25(\mathrm{OH}) \mathrm{D}$ concentration of $92.5 \mathrm{nmol} / \mathrm{L}$ [lowest (L): 80; highest (H): 145], a mean PTH of $38 \mathrm{ng} / \mathrm{L}$ (L: 18-H: 57) and a mean age of 58 years (L: 22; H: 85). The description of each subclass (men/women/menopause women) is presented in Table 3. In men, the BAP cut-off

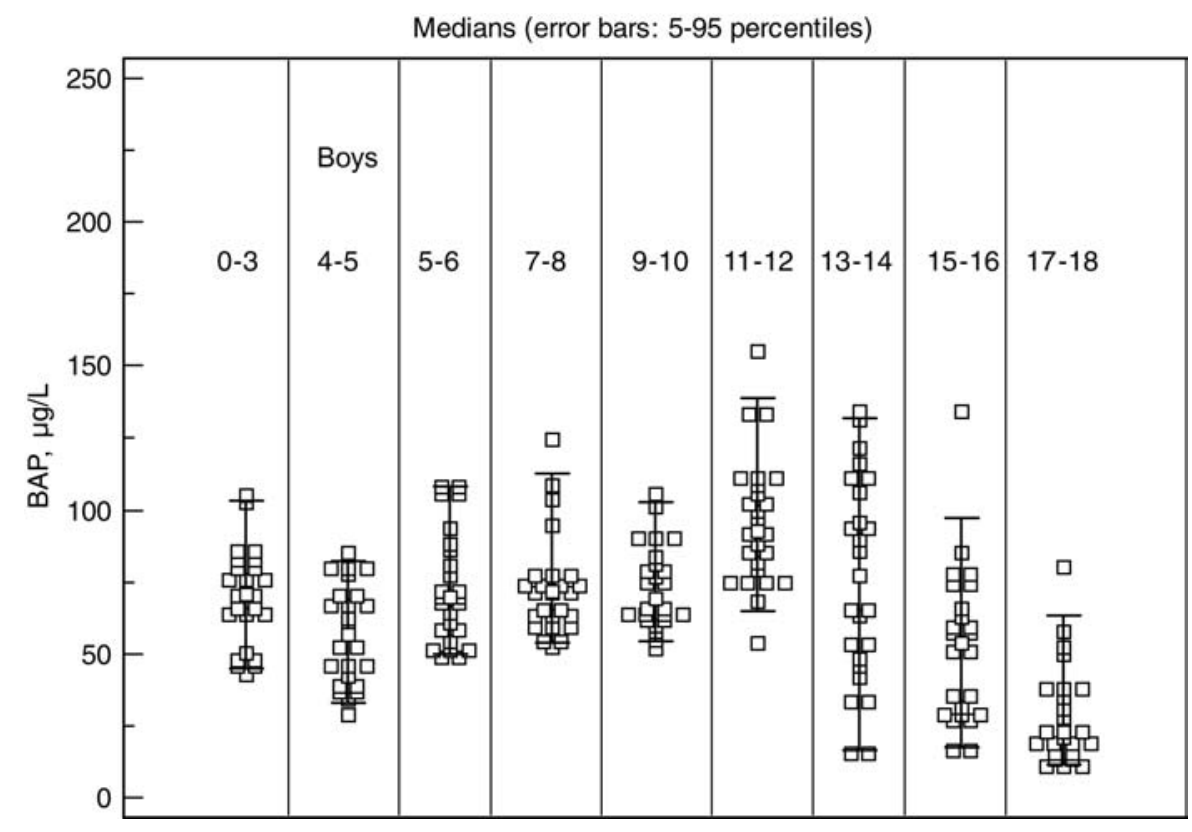

Figure 3 Age related BAP distribution in young boys. 


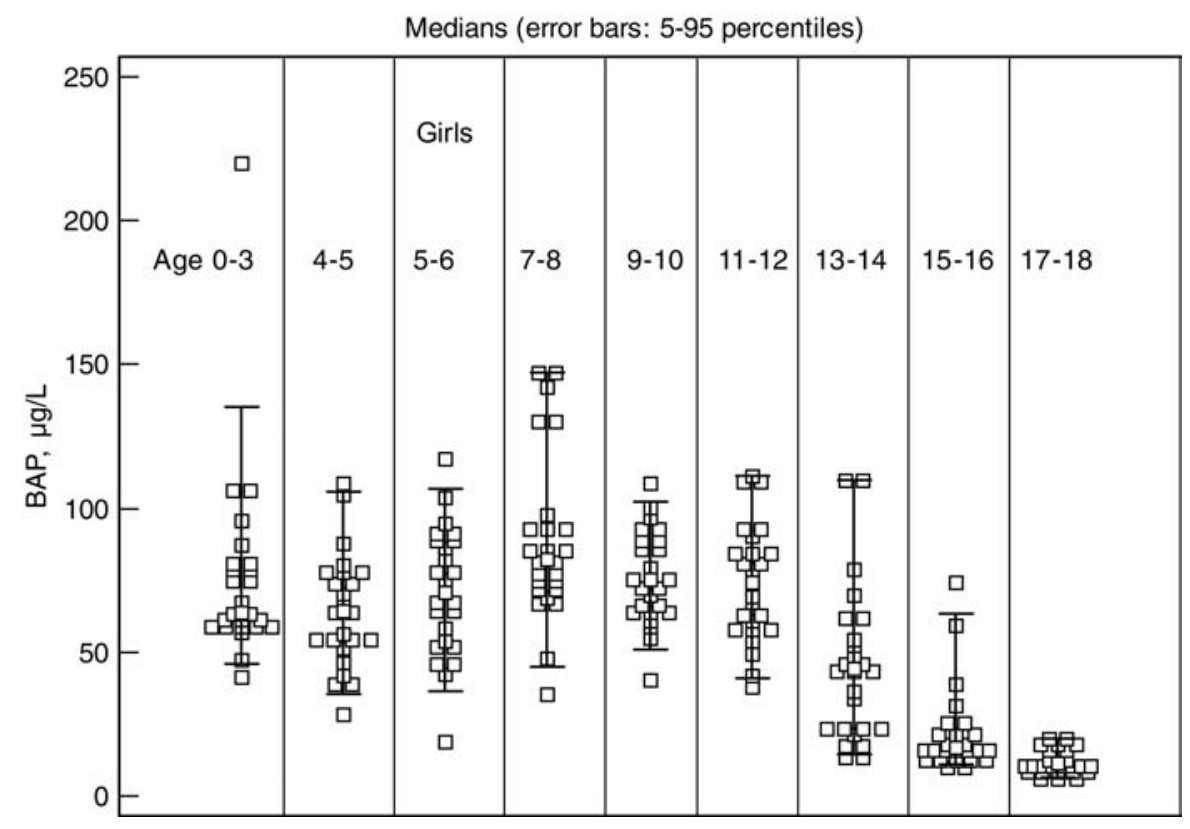

Figure 4 Age related BAP distribution in young girls.

was $20.3 \mu \mathrm{g} / \mathrm{L}$ (90\% CI: 16.1-25.2 $\mu \mathrm{g} / \mathrm{L}), 23.6 \mu \mathrm{g} / \mathrm{L}$ in non-menopause women (90\% CI: 16.9-26.6 $\mu \mathrm{g} / \mathrm{L})$ and $20.4 \mu \mathrm{g} / \mathrm{L}$ in menopause women (90\% CI: $17.8-25.0 \mu \mathrm{g} /$ L). There was no statistical difference between the three populations and we therefore combined all the data. The higher upper limit of normality observed in the entire population $(\mathrm{n}=360)$ was $21.3 \mu \mathrm{g} / \mathrm{L}$ (90\% CI: $18.3-24.2 \mu \mathrm{g} / \mathrm{L})$.

Figures 3 and 4 show the age-related BAP distribution among children. We observed a peak between 7 and 8 years for girls, whereas boys showed the peak to be later, between 11 and 12 years. Until 7-8 years of age, there was no significant difference between the values observed in children, whereas significant age-related differences were observed at older age. Table 4 gives the 2.5th and 97.5th percentiles observed in children, classified by age and gender. Each value higher than the upper validated range $(94 \mu \mathrm{g} / \mathrm{L})$ was obtained after dilution of the sample.

Table 4 Percentiles 2.5th and 97.5th observed when BAP was determined in young boys and girls.

\begin{tabular}{llll}
\hline Age, years & $\begin{array}{l}\text { No. in each } \\
\text { gender } \\
\text { category }\end{array}$ & $\begin{array}{l}\text { Boys, } \\
\mu \mathrm{g} / \mathrm{L}\end{array}$ & $\begin{array}{l}\text { Girls, } \\
\mu \mathrm{g} / \mathrm{L}\end{array}$ \\
\hline From 0 to $<3$ & 25 & $43.4-104.8$ & $41.9-107.0$ \\
From $\geq 3$ to $\leq 4$ & 25 & $29.7-84.8$ & $29.5-108.5$ \\
From $\geq 5$ to $\leq 6$ & 25 & $48.8-109.0$ & $21.9-115.4$ \\
From $\geq 7$ to $\leq 8$ & 25 & $52.6-123.0$ & $37.1-147.9$ \\
From $\geq 9$ to $\leq 10$ & 25 & $52.3-105.4$ & $42.0-107.6$ \\
From $\geq 11$ to $\leq 12$ & 25 & $55.7-152.3$ & $38.6-111.2$ \\
From $\geq 13$ to $\leq 14$ & 25 & $15.5-134.0$ & $13.7-109.8$ \\
From $\geq 15$ to $\leq 16$ & 25 & $16.6-127.9$ & $10.2-72.6$ \\
From $\geq 17$ to $\leq 18$ & 25 & $11.0-77.6$ & $5.9-20.0$ \\
\hline
\end{tabular}

\section{Discussion}

We present the results of the analytical validation of the Liaison BAP OSTASE. Our results show that this method is sensitive and precise. We also established the measurement uncertainty and showed that for values between 7 and 94 $\mu \mathrm{g} / \mathrm{L}, 95 \%$ of the results will be within $\pm 15 \%$ maximum allowable total error. The results of the Liaison BAP OSTASE agree with those obtained with the Beckman-Coulter Access Ostase. We established a reference range in a population that we defined as "normal", although this concept is rather controversial. We defined our reference population as adults presenting with "normal" 25(OH)D concentrations and no other causes of hyperparathyroidism (13). This approach is quite new, as these parameters are not generally taken into consideration when establishing reference ranges for bone markers (14). This point is important, as we have shown that in our defined reference population, there was no difference between males and non-menopause/menopause females. Differences are usually observed in less welldefined populations. We also provided values from 450 children classified according to age and gender. Even though our population of children cannot be considered a "reference", population, this information can be helpful to laboratories and paediatricians.

\section{Conflict of interest statement}

Authors' conflict of interest disclosure: The authors stated that there are no conflicts of interest regarding the publication of this article.

Research funding: None declared. 
Employment or leadership: None declared.

Honorarium: None declared.

\section{References}

1. Robinson R. The possible significance of hexosephosphoric esters in ossification. Biochem J 1923;17:286-93.

2. Raymond F, Datta H, Moss D. Alkaline phosphatase isoforms in bile and serum and their generation from cells in vitro. Biochim Biophys Acta 1991;1074:217-22.

3. Posen S, Grunstein HS. Turnover rate of skeletal alkaline phosphatase in humans. Clin Chem 1982;28:153-4.

4. Puche RC, Caferra DA, Rosillo I. Bone isoenzyme of serum alkaline phosphatase measured with wheat-germ agglutinin. Clin Chem 1988;34:1372-4.

5. Clinical and Laboratory Standards Institute (formerly NCCLS). Evaluation of precision performance of quantitative measurement methods; approved guideline, 2nd ed. CLSI document EP5-A2. Wayne, PA, 2004.

6. Clinical and Laboratory Standards Institute (formerly NCCLS). Evaluation of the linearity of quantitative measurement procedures: a statistical approach; approved guideline; CLSI document EP6-A. Wayne, PA, 2003:23(16).

7. Clinical and Laboratory Standards Institute (formerly NCCLS).
Evaluation of the linearity of quantitative analytical methods; proposed guideline. CLSI document. Wayne, PA, 1986.

8. EURACHEM/CITAC Guide CG 4. Quantifying uncertainty in analytical measurement, 2nd ed. 2000.

9. International Organization for Standardization (ISO). ISO/DTS 21748 Guide to the use of repeatability, reproducibility and trueness estimates in measurement uncertainty estimation. Geneva, 2003.

10. International Organization for Standardization (ISO). ISO 5725-1. Application of the statistics - accuracy (trueness and precision) of the results and methods of measurement - Part 1: general principles and definitions. Geneva, 1994.

11. Bland JM, Altman DG. Statistical methods for assessing agreement between two methods of clinical measurement. Lancet 1986;1:307-10.

12. Beck-Jensen JE, Kollerup G, Sorensen HA, Pors NS, Sorensen $\mathrm{OH}$. A single measurement of biochemical markers of bone turnover has limited utility in the individual person. Scand J Clin Lab Invest 1997;57:351-9.

13. Cavalier E, Delanaye P. Defining a "reference population": no easy task. J Bone Miner Res 2009.

14. Glover S, Gall M, Schoenborn-Kellenberger O, Wagener M, Garnero P, Boonen S, et al. Establishing a reference interval for bone turnover markers in 637 healthy, young, pre-menopausal women from UK, France, Belgium and the USA. J Bone Miner Res 2008 